\title{
Computação Plugada Ordenação: Um Aplicativo para Ensinar sobre Algoritmos de Ordenação na Educação Básica
}

\author{
Reinaldo da Veiga Lima, Ayla Dantas Rebouças \\ Universidade Federal da Paraíba - UFPB \\ Campus IV - Rio Tinto - PB \\ $\{r e i n a l d o \cdot v e i g a$, ayla\}@dcx.ufpb.br
}

\begin{abstract}
This work aims to present "Computação Plugada Ordenação", an Android application developed with the goal of teaching basic computing concepts related to sorting algorithms for children (8+ years) and young people. The current version is based on the activity of sorting algorithms from the unplugged computing book by Tim Bell et al. and explores the Quicksort and Insertion Sort algorithms. To evaluate the first prototype, online questionnaires were made available to teachers and alumni in the areas of computing and education, and an online questionnaire was also made available to potential users. Good acceptance was observed from the first assessment. Some suggestions for improvements were related to the colors and letter sizes used.
\end{abstract}

Resumo. $O$ presente trabalho tem como objetivo apresentar o aplicativo "Computação Plugada Ordenação", um aplicativo Android desenvolvido com o intuito de ensinar conceitos básicos da computação relacionados a algoritmos de ordenação para crianças com 8 anos ou mais e jovens. A versão atual é baseada na atividade de algoritmos de ordenação do livro de computação desplugada de Tim Bell e outros autores e explora os algoritmos Quicksort e Insertion Sort. Para avaliar o primeiro protótipo foram disponibilizados questionários online para professores e licenciados das áreas de computação e educação e também foi disponibilizado um questionário online para possíveis usuários. Foi observada uma boa aceitação nessa primeira avaliação. Algumas sugestões de melhoria dadas se referiam às cores e tamanho das letras utilizadas.

\section{Introdução}

Vivemos em uma sociedade onde a tecnologia está presente em diversos setores. Por exemplo, o uso da tecnologia no ambiente educacional está crescendo cada vez mais e rompendo paradigmas na forma de ensinar e aprender [Delfino, Rebouças e Meireles 2016]. De acordo com Bell et al. (2011), o ensino de conceitos computacionais com o uso da computação desplugada auxilia no desenvolvimento de habilidades de comunicação, resolução de problemas, criatividade e cognição num contexto significativo.

A introdução ao pensamento computacional na educação básica é de suma importância, pois além de possibilitar aos alunos adquirirem conhecimentos na área de informática, o pensamento computacional auxilia os estudantes na resolução de problemas relacionados ao seu cotidiano e nas matérias escolares, melhorando o rendimento escolar [Lima e Sousa 2015]. Diante disso, de acordo com Wangenheim et al. (2019), "fomentar o pensamento computacional já desde a educação básica parece 
desejável, preparando as crianças para as demandas atuais e futuras de nossa sociedade do conhecimento".

Desta forma, mostra-se evidente que o ensino de conceitos da computação e do pensamento computacional pode ser de muita importância para a educação básica. Diante disso, a ferramenta elaborada neste trabalho busca ensinar um dos conceitos básicos da computação, que são algoritmos de ordenação, para estudantes da educação básica. Isso é feito transformando em aplicativo para dispositivos móveis a atividade de algoritmos de ordenação do livro de computação desplugada de Bell et al. (2011). Conforme destacado em um site da UNICAMP ${ }^{1}$ sobre essa atividade, em sua versão original ela favorece o desenvolvimento de diferentes habilidades e competências sugeridas na Base Nacional Comum Curricular - $\mathrm{BNCC}^{2}$ e relacionadas à ordenação de números naturais.

Por meio de um aplicativo interativo construído com base em atividade da computação desplugada, busca-se neste trabalho destacar a importância de conhecer sobre algoritmos e em especial os de ordenação, além de apresentar conceitos e atividades relacionadas a algoritmos de ordenação de maneira atrativa para crianças e jovens, visando disseminar as atividades propostas por Bell et al. (2011). Este trabalho é uma evolução dos trabalhos de Oliveira (2019) e Gomes Neto (2019), os quais tinham como foco um aplicativo referente à atividade desplugada sobre números binários e que visava disseminar esta atividade. Parte do aplicativo de binários desenvolvido é descrito no artigo de Oliveira, Dantas e Gomes Neto (2019). Esse aplicativo foi chamado Computação Plugada e permite que os usuários consigam individualmente realizar exercícios recebendo feedback automático.

No trabalho de Oliveira (2019), atualizado e ampliado no artigo de Oliveira e Rebouças (2021), foi apresentada uma avaliação da primeira versão do aplicativo Computação Plugada por meio de disponibilização de questionários online para estudantes e para professores de computação que já possuíam experiência com computação desplugada. Esta avaliação teve resultados positivos que dão indícios de que o aplicativo pode ser adequado para utilização individual ou em sala de aula. No trabalho de Gomes Neto (2019) é apresentada uma nova versão de interface para o aplicativo anterior e com foco no público infantil (crianças na faixa etária de 7-9 anos de idade). $\mathrm{O}$ aplicativo foi chamado de Computação Plugada Kids e essa proposta inicial de interface foi avaliada por especialistas. Posteriormente, esse aplicativo tratado nos trabalhos foi renomeado para "Computação Plugada Binários" e novos aplicativos foram sendo propostos pelo projeto "Computação Plugada"3 para "plugar" outras atividades desplugadas do livro de Bell et al. (2011), como é o caso do aplicativo "Pixel" [Figueiredo et al.2021], que trata da atividade "Colorindo com Números - Representação de Imagens" e do presente trabalho, que apresenta o aplicativo "Computação Plugada Ordenação", que foca na atividade "O mais leve e o mais pesado-Algoritmos de Ordenação".

Para realizar uma avaliação inicial do aplicativo "Computação Plugada Ordenação" foram disponibilizados dois questionários online construídos com base nos que foram utilizados por Oliveira e Rebouças (2021). Um desses questionários ${ }^{4}$ destinava-

\footnotetext{
${ }^{1} \mathrm{http} / / /$ desplugada.ime.unicamp.br/

${ }^{2} \mathrm{http}: / /$ basenacionalcomum.mec.gov.br/images/BNCC_EI_EF_110518_versaofinal_site.pdf

${ }^{3} \mathrm{https}: / /$ sites.google.com/view/computacaoplugada/

${ }^{4}$ https://forms.gle/W583JpQYVveNkPt5A
} 
se a professores e licenciados das áreas de computação e educação e outro era destinado a possíveis usuários do aplicativo. Achamos necessário realizar uma avaliação inicial com profissionais da computação e educação para verificar se consideravam corretos e apropriados os conceitos apresentados no aplicativo e sua percepção sobre a aplicabilidade da solução na educação básica, além de podermos avaliar outras questões como usabilidade e possíveis sugestões de melhoria. No caso do segundo questionário ${ }^{5}$, buscou-se obter a percepção de aprendizagem de possíveis usuários do aplicativo sob o ponto de vista de alguém que o utiliza para aprender, além de avaliar a aceitação de maneira geral. Embora o público-alvo do aplicativo fossem alunos da educação básica, alunos do ensino superior também o responderam e forneceram feedbacks importantes para a evolução do aplicativo.

Este artigo está organizado conforme descrito a seguir. Na Seção 2 é apresentada a contextualização sobre algoritmos de ordenação, apresentando os algoritmos de ordenação e algumas ferramentas para apoiar o ensino deste conteúdo. Na Seção 3 é apresentada a metodologia para o desenvolvimento da solução apresentada. Na Seção 4 se encontra a apresentação do aplicativo. A Seção 5 apresenta os resultados com base nos dados qualitativos e quantitativos obtidos a partir da aplicação dos questionários. Na Seção 6 podemos encontrar as conclusões e propostas de trabalhos futuros.

\section{Ensino de Algoritmos de Ordenação}

O ensino de algoritmos de ordenação é de suma importância, pois sem o uso da ordenação seria mais difícil realizar tarefas que envolvem a localização de uma informação. Além de facilitar a procura de informações, a ordenação auxilia na identificação de valores mais altos e mais baixos. É importante também destacar que o ensino de algoritmos de ordenação auxilia na escolha e identificação de métodos de ordenação mais rápidos e eficientes, desta forma fazendo com que se gaste menos tempo para ordenar as coisas [Bell et al. 2011].

Existem vários tipos de algoritmos de ordenação. Entre eles estão: ordenação por inserção (insertion sort) e ordenação rápida (quicksort). Conforme apresentado por Bell et al. (2011), quando utilizamos o algoritmo "insertion sort", nós vamos removendo cada objeto de um grupo desordenado e o inserimos na sua posição correta em uma lista ordenada. A cada inserção, o grupo de objetos desordenados diminui e a lista ordenada aumenta até que, finalmente, toda a lista esteja ordenada. O método "quicksort" é apresentado por Bell et al. (2011) como uma forma de ordenar onde se deve escolher um dos objetos de forma aleatória. Em seguida, devemos comparar cada um dos objetos restantes com ele. Colocamos então aqueles que são menores do lado esquerdo, e os maiores à direita do objeto escolhido que fica no meio. Posteriormente, devemos escolher um dos grupos de objetos e repetir esse procedimento para cada um dos grupos. Embora existam outras fontes que abordem estes algoritmos, como Cormen et al. (2012), o livro de Bell et al. (2011) foi escolhido como fonte principal pela forma didática e lúdica como o apresenta e pelo foco do projeto Computação Plugada, no qual se inseriu este trabalho, em transformar em aplicativos as atividades ali descritas, fazendo as devidas adaptações. No livro, por exemplo, foca-se em pesos para fazer a ordenação dos itens mais leves e mais pesados, mas no aplicativo proposto neste trabalho decidiu-se utilizar cartas nas ordenações.

\footnotetext{
${ }^{5}$ https://forms.gle/R1GdhNycqLforibV9
} 
Existem diversas ferramentas para ensino-aprendizagem de algoritmos de ordenação. Por exemplo, Veras et al. (2010) apresentam em seu trabalho uma ferramenta computacional para o ensino de algoritmos de ordenação chamada SEED. Esta ferramenta provê meios de estudo e comparação de algoritmos de ordenação de dados Insertion Sort, Selection Sort, BubbleSort, MergeSort, HeapSort e QuickSort para alunos da área de computação e pode ser utilizada como ferramenta de ensino-aprendizagem nas disciplinas "Estruturas de Dados" e "Projeto e Análise de Algoritmos". Para o desenvolvimento do SEED, foram utilizadas a linguagem de programação Java e o ambiente de desenvolvimento NetBeans.

Battistella, Wangenheim e Wangenheim (2012) desenvolveram um jogo para ensino de algoritmos de ordenação chamado SORTIA, onde o aluno deve simular manualmente o processo de ordenação de um conjunto de números inteiros. O SORTIA tem como intuito apresentar uma forma alternativa ao ensino tradicional dos algoritmos na disciplina de Estrutura de Dados. Para o desenvolvimento do jogo foram utilizadas as tecnologias JavaScript e HTML. Nunes e Parreira Júnior (2015), por sua vez, apresentaram um jogo denominado RPG4Sorting, que é um jogo educacional para auxílio ao ensino de métodos de ordenação. Este jogo de tabuleiro, que pode ser aplicado em salas de aula convencionais, tem o intuito de aprimorar a interação entre alunos e professores durante o processo de ensino-aprendizagem de métodos de ordenação visando um aprendizado colaborativo.

Delfino, Rebouças e Meireles (2016) apresentaram em seu trabalho uma ferramenta para o ensino-aprendizagem de algoritmos de ordenação e pesquisa, com o intuito de que alunos da disciplina de Computação e Algoritmos compreendessem o funcionamento dos algoritmos de forma rápida e dinâmica. $\mathrm{O}$ aplicativo permite a visualização do código fonte do algoritmo na linguagem de programação Java. Além disso, demonstra o passo a passo do algoritmo de acordo com os números passados pelo usuário, sendo assim uma forma dinâmica de demonstrar o algoritmo. A tecnologia escolhida para o desenvolvimento do aplicativo foi o Android. Já no trabalho de Johansson e Flor (2018) é apresentada uma ferramenta gráfica para o ensino de algoritmos de ordenação chamada KLOSS. Esta ferramenta apresenta animações dos métodos de ordenação Bubble Sort, Insertion Sort e Quick Sort. Kloss foi desenvolvida no ambiente Scratch e foi empregada na disciplina Algoritmos e Linguagem de Programação II, em um Curso Técnico em Informática Integrado ao Ensino Médio. Um outro trabalho relacionado, embora não diretamente associado a algoritmos de ordenação é o de Werlich et al. (2019), onde é apresentado um estudo de caso realizado com dois objetos de aprendizagem para ordenação alfabética, um plugado e outro desplugado, na disciplina Pensamento Computacional em uma escola particular de Ensino Fundamental I.

A principal diferença entre os trabalhos citados anteriormente e este trabalho, é que o "Computação Plugada Ordenação" foi criado com base em uma atividade do livro computação desplugada [Bell et al. 2011], sobre algoritmos de ordenação e tem como público-alvo estudantes da educação básica (o que não é o foco de todos os trabalhos apresentados), além de se focar em dois algoritmos de ordenação: o Quicksort e o Insertion Sort. Tal escolha foi feita durante o processo de concepção do aplicativo como forma de explorar um algoritmo que fosse fácil de ser entendido por crianças e jovens explorando cartas (o Insertion Sort) e um algoritmo através do qual pudéssemos apresentar de maneira simplificada o conceito de recursão (o Quicksort). É importante destacar que não foi encontrado nenhum outro trabalho com foco no ensino de algoritmos 
de ordenação a partir da busca realizada com a chave "ordenação" por meio do portal br.ie nos anais do SBIE entre os anos de 2001 e 2020 e do WIE entre os anos de 2003 e 2020, e nem nas buscas nos anais do WEI entre os anos de 2014 e 2020 consultados pelo Portal Sol da SBC.

\section{Metodologia para Desenvolvimento da Solução}

As bases teóricas sobre pensamento computacional para a realização deste trabalho foram obtidas por meio de pesquisa bibliográfica e por meio da análise de trabalhos anteriores, principalmente os que foram produzidos pelo projeto Computação Plugada ${ }^{6}$, que eram os mais relacionados. Analisando atividades do livro de Bell et al. (2011) que ainda não tinham sido "plugadas", decidimos escolher a atividade relacionada a algoritmos de ordenação no intuito de tentar construir um aplicativo que apresentasse o conceito de algoritmos de forma prática e lúdica. Iniciou-se então uma busca na literatura por conceitos e ferramentas de ensino de algoritmos de ordenação para assim obter melhor embasamento na construção de uma ferramenta de ensino de algoritmos de ordenação para crianças e jovens e ter uma ideia de ferramentas já construídas.

Posteriormente, foram definidas as ferramentas necessárias para a construção do aplicativo e para a sua prototipagem de telas. Para a implementação foi utilizado o ambiente de desenvolvimento Visual Studio Code e o React Native e decidimos focar na plataforma Android, assim como vinha sendo adotado para a construção de outros aplicativos do projeto Computação Plugada. Segundo Eisenman (2015), o React Native é um framework JavaScript que permite a construção de aplicações nativas para os sistemas iOS e Android. O React Native ${ }^{7}$ visa tornar o processo de desenvolvimento mais fácil e possibilita a criação de aplicações para as plataformas Android e iOS. Além disso, o React Native permite que seu aplicativo use as mesmas APIs da plataforma nativa que outros aplicativos usam.

Para o desenvolvimento foi utilizada a linguagem de programação JavaScript, que é uma linguagem de programação que apresenta características dinâmicas e que tem como objetivo principal oferecer melhor interatividade às páginas construídas [Oliveira 2020]. Antes de iniciar o desenvolvimento em si e após o levantamento de informações sobre os conteúdos a trabalhar no aplicativo, foi realizada a prototipagem de telas utilizando a ferramenta web Figma ${ }^{8}$, que é uma ferramenta de design online que auxilia a colaboração em equipe e facilita a comunicação e o feedback. O Figma foi utilizado para a criação do protótipo das telas da aplicação visando uma melhor orientação para o desenvolvimento do aplicativo e evitando retrabalho no desenvolvimento. Após a definição e reuniões de discussão sobre cada nível do aplicativo, era feita a implementação do nível e voltava-se à prototipagem das telas do nível seguinte. A prototipagem e o desenvolvimento do aplicativo foram feitos com base nas pesquisas bibliográficas e estudos realizados sobre as tecnologias utilizadas e códigos pré-existentes referentes ao aplicativo Computação Plugada - Binários disponível no GitHub 9 .

\footnotetext{
${ }^{6} \mathrm{https}: / /$ sites.google.com/view/computacaoplugada/

${ }^{7}$ https://reactnative.dev

${ }^{8} \mathrm{https}$ ://www.figma.com/organization/

${ }^{9}$ https://github.com/pluggedcomputing/binary-numbers
} 
Após a finalização da construção do primeiro protótipo do aplicativo, que contava com 4 níveis, que serão apresentados na Seção 4, foi realizada uma avaliação inicial de caráter quanti-qualitativa [Souza;Kerbauy 2017] do aplicativo junto a professores, licenciados das áreas de computação e educação e também com possíveis usuários. Essa avaliação foi feita através do levantamento de dados por meio de questionários online elaborados no Google Forms ${ }^{10}$. Foram produzidos dois questionários, os quais encontramse disponíveis em https://forms.gle/W583JpQYVveNkPt5A e https://forms.gle/R1GdhNycqLforibV9. Tais questionários foram construídos com base nos que foram utilizados no trabalho de Oliveira e Rebouças (2021) e o propósito de cada um foi apresentado na introdução deste trabalho. Os dois questionários foram divulgados através da lista de e-mails da graduação dos cursos de Licenciatura em Ciência da Computação e de Bacharelado em Sistemas de Informação da UFPB - Campus IV e também foram enviados para alguns contatos e grupos via mensagens de WhatsApp. Os resultados do levantamento estão apresentados na Seção 5.

\section{O Aplicativo Computação Plugada - Ordenação}

O aplicativo "Computação Plugada Ordenação" visa ensinar conceitos básicos de algoritmos de ordenação, mas sem utilizar linguagens de programação, desta forma buscando um aprendizado mais intuitivo até para pessoas que não entendem da área ou que não dispõem de computadores para programar.

O código fonte do aplicativo pode ser encontrado através do endereço https://github.com/reinaldoveiga/sorting algorithms.git. Ele é um aplicativo para dispositivos Android e foi implementado no ambiente de desenvolvimento Visual Studio Code, utilizando-se a ferramenta React Native e a linguagem de programação JavaScript, como apresentado anteriormente. A primeira versão (1.0) do aplicativo, que foi a utilizada neste trabalho, pode ser baixada no endereço https://drive.google.com/drive/folders/1oY7PbRFcHscSpXgiBWywN2j-

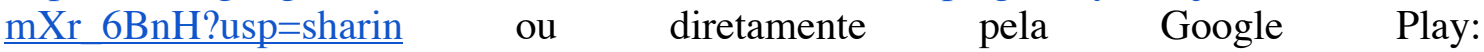
https://play.google.com/store/apps/details?id=br.ufpb.dcx.computacaoplugada.sortingal gorithms. O aplicativo também possui um vídeo de apresentação da sua execução que está disponível em: https://youtu.be/YNb84QvMc3Y.

Para a primeira versão do aplicativo "Computação Plugada Ordenação" foi escolhida a atividade "O mais leve e o mais pesado-Algoritmos de Ordenação" da segunda parte do livro de Bell et al. (2011), denominada "Colocando os Computadores para Trabalhar-Algoritmos". Essa atividade aborda como os computadores são utilizados para colocar listas em ordem através da utilização de métodos de ordenação e destaca a importância da utilização de métodos inteligentes para executar tarefas rapidamente. Segundo o próprio livro, a atividade pode ser aplicada para crianças a partir de 8 anos e está correlacionada à disciplina de matemática, desenvolvendo as habilidades de ordenar e comparar. Por meio do aplicativo, através de atividades interativas, o usuário pode ver como funcionam os métodos de ordenação e obter feedback automático sobre seus erros e acertos ao ordenar números dispostos em cartas.

O aplicativo em questão não está utilizando números acima de uma dezena para que possa também contemplar crianças a partir de 8 anos, desta forma facilitando a

\footnotetext{
${ }^{10} \mathrm{https}: / / \mathrm{www}$.google.com/forms/about/
} 
assimilação do conteúdo abordado no aplicativo. Entretanto, o aplicativo em suas futuras versões pode ser evoluído para contemplar números maiores nos exercícios apresentados.

A versão utilizada neste trabalho conta com 4 níveis, detalhados no Quadro $1 \mathrm{e}$ segue a mesma ideia geral de níveis do aplicativo "Computação Plugada Binários". Em cada nível são apresentadas explicações e atividades que foram baseadas no conteúdo do livro de Bell et al. (2011), com adaptações, como o uso de cartas ao invés de pesos no caso da atividade escolhida. O processo de construção e definição dos níveis se deu de forma iterativa e incremental envolvendo discussões, um processo de design em dupla e pesquisas dos autores deste trabalho visando a criação de um aplicativo que pudesse apresentar de forma simples e atrativa a noção de algoritmos, do conceito de recursão e também de algoritmos de ordenação em si. Para isso, e visando não sobrecarregar os estudantes em um contato inicial com a área, decidimos focar em dois algoritmos de ordenação e consideramos que o uso do algoritmo de inserção (Insertion Sort) e do QuickSort seria apropriado após prototipagens iniciais das telas.

Quadro 1. Descrição dos níveis implementados na versão 1.0 do aplicativo

\begin{tabular}{|l|l|}
\hline \multicolumn{1}{|c|}{ Nível } & \multicolumn{1}{c|}{ Descrição } \\
\hline $\begin{array}{l}\text { Nível 1 - } \\
\text { Introdução }\end{array}$ & $\begin{array}{l}\text { Nesse nível são apresentadas informações sobre o uso de computadores para } \\
\text { ordenação e sobre a importância de dados ordenados. Ao final desse nível, é } \\
\text { apresentada uma atividade de múltipla escolha relacionada ao que é apresentado } \\
\text { inicialmente. }\end{array}$ \\
\hline $\begin{array}{l}\text { Nível 2 - } \\
\text { Ordenação por }\end{array}$ & $\begin{array}{l}\text { É apresentado nesse nível o método de ordenação por Inserção (Insertion Sort) por } \\
\text { meio de exemplos e exercícios com cartas a serem ordenadas. Após responder } 2 \\
\text { questões de múltipla escolha, o usuário é convidado a assistir um vídeo de menos de 3 } \\
\text { minutos com mais detalhes sobre esse tipo de ordenação e um exemplo passo a passo. }\end{array}$ \\
\hline $\begin{array}{l}\text { Nível 3 - } \\
\text { Quicksort }\end{array}$ & $\begin{array}{l}\text { São apresentadas nesse nível as ideias básicas sobre o método de ordenação rápida } \\
\text { (Quicksort) e questões de múltipla escolha por meio das quais vão sendo ordenadas } \\
\text { cartas utilizando o método. Após responder as questões, o usuário é convidado a ver } \\
\text { um vídeo de menos de 3 minutos sobre esse tipo de ordenação na prática. }\end{array}$ \\
\hline $\begin{array}{l}\text { Nível 4 - } \\
\text { Comparação } \\
\text { dos } \\
\text { Algoritmos }\end{array}$ & $\begin{array}{l}\text { Apresenta por meio de um vídeo de menos de 4 minutos a comparação dos } \\
\text { algoritmos de ordenação abordados no aplicativo, possibilitando ao aluno refletir } \\
\text { sobre o tempo e quantidade de comparações necessárias ao utilizar cada método de } \\
\text { ordenação. Após assistir o vídeo, o usuário deve responder questões de múltipla } \\
\text { escolha sobre o conteúdo abordado. Além disso, são apresentados por meio de textos } \\
\text { ilustrados os conceitos de algoritmos, de recursão e de divisão e conquista e sua } \\
\text { relação com os métodos de ordenação vistos. }\end{array}$ \\
\hline
\end{tabular}

$\mathrm{Na}$ Figura 1 apresentada a seguir podemos observar algumas das telas do aplicativo. Na tela ilustrada pela Figura 1a, que faz parte do nível 1 do aplicativo, o usuário verá uma explicação sobre a importância de ordenar. Na Figura 1b é ilustrada uma tela contendo uma questão de múltipla escolha sobre o conteúdo abordado. Na Figura 1c está ilustrada uma das telas exibidas quando o usuário completa cada nível. Nessa tela aparece uma mensagem de parabéns, informando o nível completado e ofeedback relativo ao que se espera que o usuário conheça ao concluir cada nível. A cada nível finalizado, o próximo será liberado para o usuário até que finalize todos os níveis. A Figura 1d ilustra uma das telas de exibições de vídeos onde o usuário pode executar, pausar a execução do vídeo, voltar ou adiantar. Ele pode ainda aumentar ou diminuir o volume e clicar no botão "Já Assisti" para seguir para a próxima tela do aplicativo. 


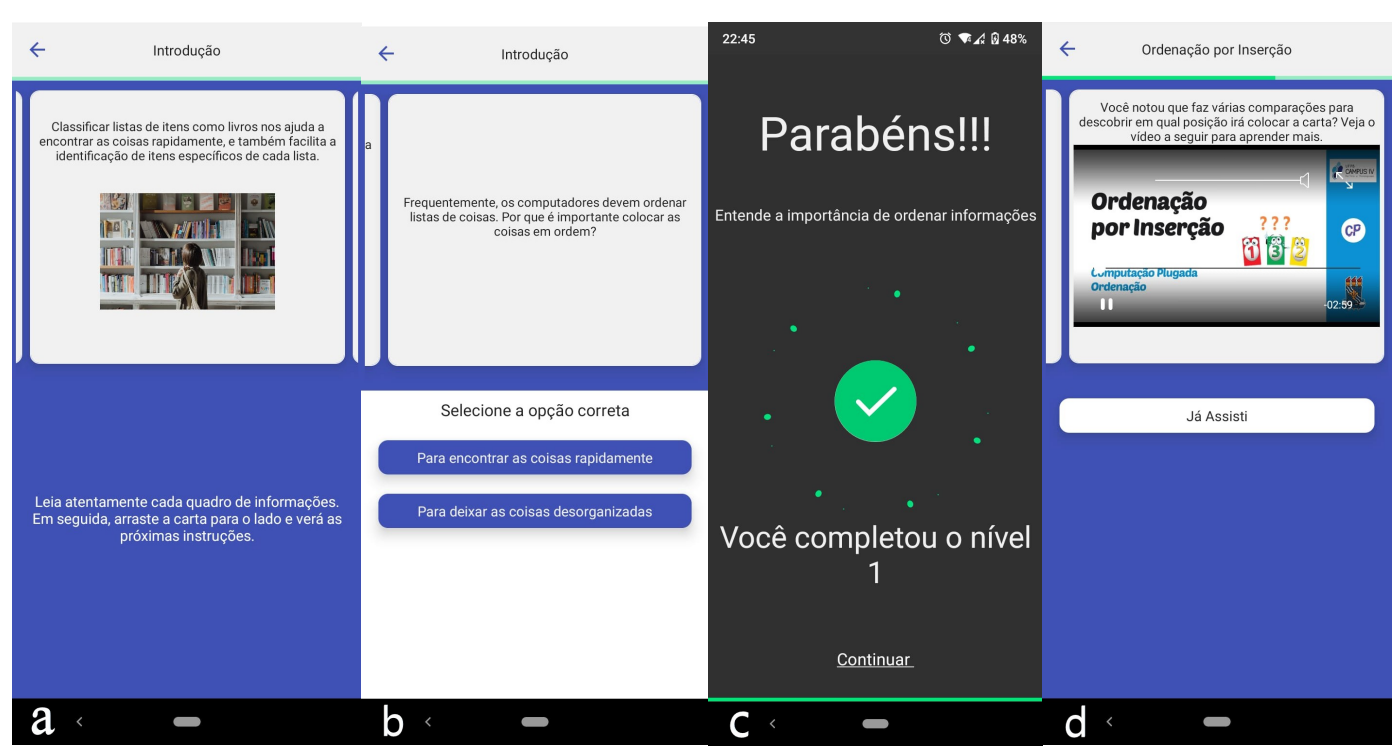

Figura 1. Tela de explicação (a). Tela de exercício (b). Tela de feedback do Nível 1 (c). Tela de exibição de vídeo (d).

\section{Resultados de Avaliações do Aplicativo}

\subsection{Avaliação Realizada por Professores ou Licenciados}

A avaliação aplicada por meio de um questionário a professores e licenciados da área de computação e educação buscava coletar informações sobre os participantes (como sua formação e seu nível de familiaridade com o conteúdo algoritmos de ordenação) e também buscava coletar impressões sobre os conceitos de algoritmos de ordenação apresentados no aplicativo e a opinião dos participantes quanto à possível utilização do aplicativo "Computação Plugada Ordenação" como ferramenta de ensino de algoritmos de ordenação para alunos da educação básica. Foram obtidas 6 respostas, sendo 4 de professores de computação (P1, P4, P5, P6) e dois (P2 e P3) que não são da área. Dentre os participantes, 5 tinham formação (curso superior, licenciatura ou pós-graduação) em áreas da computação (P1, P2, P4, P5 e P6) e 1 tinha formação na área da educação (P3). Dentre eles, apenas 1 possui experiência em ensino na educação básica (P3). Dos participantes, 4 eram homens (P1, P2, P4 e P6) e 2 mulheres (P3 e P5) e suas idades variaram entre 25 e 42 anos.

Para realizar a avaliação, 4 dos participantes (P1, P2, P5 e P6) instalaram o aplicativo e completaram todos os níveis e 2 participantes (P3 e P4) fizeram a avaliação com base em um vídeo demonstrando todos os níveis do aplicativo e disponível em: https://youtu.be/YNb84QvMc3Y. Sobre o ensino de algoritmos de ordenação em alguma disciplina, 4 professores (P1, P4, P5 e P6) já ensinaram algoritmos de ordenação em disciplina do ensino superior, 1 (P3) já ensinou em disciplina da educação básica e 1 não ensinou (P2), porém conhece o conteúdo algoritmos de ordenação.

De acordo com os participantes que responderam este questionário, todos consideraram que os conceitos de algoritmos de ordenação apresentados no aplicativo estão corretos e apropriados. No questionário, perguntou-se se achavam que o aplicativo poderia apoiar na compreensão de algoritmos de ordenação por parte de estudantes da educação básica e todos responderam que achavam que sim. Ao serem perguntados sobre 
a chance de indicar o aplicativo para estudantes da educação básica em uma escala de 0 (Nenhuma) a 10 (Muito alta), 4 participantes (P1, P2, P3 e P5) indicaram como sendo de 10 essa chance e 2 (P4 e P6) indicaram como sendo de 8 , o que mostra que em sua visão o aplicativo pode ser apropriado para estes estudantes. Ao serem questionados sobre o quanto gostaram do aplicativo em uma escala de 0 (Não gostei de forma alguma) a 10 (Adorei), 1 pessoa escolheu 8 nessa questão, 2 escolheram 9 e 3 escolheram 10.

Com relação aos pontos positivos e sugestões de melhoria indicados, alguns deles sugeriram melhorias na aparência do aplicativo, em suas cores e na apresentação dos textos utilizados (utilização de letras maiores). Foi também reportado um problema que pode se referir a algum defeito no Nível 3 do aplicativo. De maneira geral, pelas respostas obtidas, acredita-se que os participantes se mostraram satisfeitos com o aplicativo "Computação Plugada Ordenação".

\subsection{Avaliação Realizada por Usuários}

O questionário disponibilizado aos possíveis usuários do aplicativo foi aplicado a fim de obter informações sobre a sua usabilidade e percepção de aprendizagem quanto ao conteúdo algoritmos de ordenação após a utilização do aplicativo, além de sugestões de melhorias. Foram obtidas 14 respostas, sendo 10 homens e 4 mulheres, com idades entre 13 e 29 anos. Dentre os usuários que responderam o questionário 57,1\% são estudantes de computação ou áreas afins, 14,2\% são estudantes de outros cursos, 35,7\% estudantes do ensino médio e $7,1 \%$ estudantes do ensino fundamental. Além disso, 78,5\% dos participantes relataram ter completado pelo menos a metade de todos os níveis do aplicativo.

Dentre os usuários que responderam o questionário, 57,1\% nunca estudaram algoritmos de ordenação, por isso relataram que não conheciam suficientemente o conteúdo algoritmos de ordenação. 28,6\% relataram que já estudaram algoritmos de ordenação em disciplinas do ensino superior, 14,3\% estudaram o conteúdo em disciplinas da educação básica e apenas $7,1 \%$ não estudaram algoritmos de ordenação em nenhuma disciplina, porém já tinham conhecimento sobre o assunto.

Ao serem questionados sobre o quanto o aplicativo contribuiu para o seu aprendizado sobre algoritmos de ordenação em uma escala de 1 (Contribuiu Pouco) a 5 (Contribuiu Muito), a maioria das respostas foi positiva, pois apenas 1 participante apresentou resposta neutra, mas viu-se que este participante não chegou a completar nenhum nível do aplicativo por sua resposta à questão 6 do questionário. Para os demais, viu-se que 4 participantes escolheram 4 na escala (1-5) e os 9 restantes escolheram 5. Analisando as respostas, pudemos observar que o aplicativo auxiliou tanto quem não tinha nenhum conhecimento sobre algoritmos de ordenação quanto aqueles que já tinham, já que se mencionou que este lhes ajudou a relembrar o assunto.

Quanto à usabilidade do aplicativo, em uma escala de 0 (Muito difícil) a 5 (Muito fácil), 71,4\% dos usuários que participaram da pesquisa responderam que o aplicativo é muito fácil de ser utilizado (Item 5 da escala) e apenas 4 escolheram o item 4 da escala, o que foi um resultado muito positivo. Dentre as sugestões de melhoria quanto à aparência do aplicativo, algumas foram referentes ao tamanho e apresentação dos textos ou às cores utilizadas. De maneira geral, foram obtidos vários feedbacks positivos relatando que o aplicativo possui ótima aparência. Foi reportado um problema de desempenho do aplicativo referente a um erro durante a sua execução e que pretendemos investigar e 
resolver no futuro. Por fim, foi observado que de maneira geral os usuários se mostraram satisfeitos com o aplicativo. Em uma escala de 0 (Nenhuma) a 10 (Muito alta), 71,4\% escolheram os números 9 ou 10,1 escolheu 8 e 2 escolheram 7. Apenas um usuário fez uma avaliação baixa (3) neste item e talvez o que possa ter influenciado sua escolha tenha sido o fato de ter sido o único a utilizar o aplicativo pelo PC (provavelmente por emulador) ao invés de diretamente por meio de dispositivo móvel. No entanto, é importante também realizar estudos com mais estudantes da educação básica para fortalecer os resultados encontrados até então e que não podem ser generalizados dado o tamanho da amostra utilizada.

\section{Conclusões e Trabalhos Futuros}

Neste trabalho" foi apresentado o aplicativo "Computação Plugada Ordenação" que foi construído com o intuito de facilitar a aplicação da atividade de computação desplugada [Bell et al. 2011] sobre algoritmos de ordenação para crianças e jovens. No trabalho em questão foram apresentadas algumas de suas telas, seu processo de concepção e desenvolvimento, descrições dos níveis atuais do mesmo e as ferramentas que foram utilizadas para a sua implementação. Foi também apresentado o resultado de uma primeira avaliação através de questionários online.

Pode-se concluir que os resultados da avaliação do aplicativo "Computação Plugada Ordenação" foram bastante positivos e que os participantes da avaliação de maneira geral consideraram que o aplicativo pode ser útil para o ensino de algoritmos de ordenação para estudantes da educação básica. No entanto, é importante envolver em avaliações futuras mais professores e estudantes atuando nesse nível da educação e realizar algumas melhorias quanto à aparência, aos textos utilizados e possíveis defeitos relatados durante a execução do aplicativo. Embora o estudo inicial tenha sido útil no sentido de verificar os conteúdos apresentados junto a profissionais e licenciados das áreas de computação e educação e para se verificar a percepção de aprendizagem junto a possíveis usuários do aplicativo, é importante também no futuro realizar uma avaliação sobre como o aplicativo seria utilizado em uma sala de aula convencional por professor da educação básica. No futuro, pretende-se investigar a adição de mais métodos de ordenação e evoluir o aplicativo para contemplar números maiores. Pretende-se também adicionar atividades mais interativas e rankings com pontuação, visando assim melhor interação e maior engajamento dos usuários. Outros trabalhos futuros planejados são outras avaliações com diferentes públicos (especialmente com crianças e adolescentes entre 8 e 14 anos) a fim de coletar mais informações sobre a sua utilização e percepção de aprendizagem, além de verificarmos também a percepção de mais professores atuando na educação básica. Também como trabalhos futuros pretende-se realizar a implementação de melhorias e correções de possíveis defeitos.

\footnotetext{
${ }^{11}$ Este artigo apresenta uma síntese do trabalho de conclusão de curso de mesmo título do primeiro autor e foi desenvolvido como atividade do Grupo de Pesquisa em Computação Aplicada - Applied, como parte do projeto inscrito na Chamada n. 03/2020 Produtividade em Pesquisa PROPESQ/PRPG/UFPB, cujo código é o PVP13499-2020. Gráficos com alguns dos resultados obtidos podem ser vistos no TCC (https://drive.google.com/file/d/1FX8NYxpUFbBx9OLZR3XG5qjohPMtC_1i/view?usp=sharing).
} 


\section{Referências}

Battistella, P. E.; Wangenheim, A. von; Wangenheim, C. G. von (2012) "SORTIA - Um Jogo para Ensino de Algoritmo de Ordenação: Estudo de caso na Disciplina de Estrutura de Dados", Anais do $23^{\circ}$ Simpósio Brasileiro de Informática na Educação (SBIE 2012), ISSN 2316-6533.

Bell, T.; Witten, I. H.; Fellows, M.; Adams, R.; McKenzie, J. (2011) "Ensinando Ciência da Computação sem o uso do computador". Computer Science Unplugged ORG. Disponível em: https://classic.csunplugged.org/wpcontent/uploads/CSUnpluggedTeachers-portuguese-brazil-feb-2011.pdf.

Base Nacional Comum Curricular, BNCC: Disponível em: http://basenacionalcomum.mec.gov.br/images/BNCC_EI_EF_110518_versaofinal_si te.pdf. Acesso em: 22 jun. 2021.

Computação

Plugada.

Disponível em:<https://sites.google.com/view/computacaoplugada/> . Acesso em: 18 abr. 2021.

Cormen, T. H.; Leiserson, C. E.; Rivest, R. L.; Stein, C. (2012). Algoritmos - Teoria e Prática. 3a. ed. Rio de Janeiro: Elsevier,

Delfino, A. R. M.; Rebouças, N. P.; Meireles, A. M. R. (2016) "Ferramenta Computational De Ensino-aprendizagem De Algoritmos De Pesquisa E Ordenação", In: Encontro de Extensão, Docência e Iniciação Científica (EEDIC), 12., 2016, Quixadá. Anais... Quixadá: Centro Universitário Católica de Quixadá, 2016. ISSN: 2446-6042.

Disponível

em: $<$ http://publicacoesacademicas.unicatolicaquixada.edu.br/index.php/eedic/article/vie w/939>. Acesso em: 30 abr. 2021.

Eisenman, B. (2015) "Learning React Native". O'Reilly Media, Inc. ISBN: 9781491929001.

Gomes Neto, I. P. (2019) “O aplicativo Computação Plugada Kids”. Trabalho de conclusão de curso (Graduação em Licenciatura em Ciência da Computação) Universidade Federal da Paraíba, Rio Tinto-PB.

Google Forms. Free Online Surveys for Personal Use. Disponível em: <https://www.google.com/forms/about/>. Acesso em 27 jun. 2021.

Johansson, K. M.; Flor, D. E. (2018) "Kloss: Ferramenta Gráfica Para O Ensino De Algoritmos De Ordenação Kloss: Graphical Tool For Teaching Pf Sorting Algorithms". Revista Mundi Engenharia, Tecnologia e Gestão. Paranaguá, PR, v.3, n.4, dezembro de 2018.

Lima, Á. C.; Sousa, D. F. (2015) "Desenvolvimento do Raciocínio Lógico Algoritmo na Educação Básica”. II CONEDU Congresso Nacional de Educação.

Nunes, I. de Freitas; Parreira Júnior, P. A. (2015) "RPG4Sorting - Um Jogo Educacional para Auxílio ao Ensino de Métodos de Ordenação". Anais do XXIII Workshop Sobre Educação Em Computação (WEI).

Oliveira, M. B. (2019) “Avaliando um Aplicativo Android Para Apoiar a Aplicação de Exercícios de Computação Desplugada”. Trabalho de conclusão de curso (Graduação 
em Licenciatura em Ciência da Computação) - Universidade Federal da Paraíba, Rio Tinto-PB.

Oliveira, M. B.;, Dantas, A.; Gomes Neto, I. P. (2019) “Computação Plugada: Um Aplicativo Android Para Apoiar a Aplicação de Exercícios de Computação Desplugada". In Anais do XXVII Workshop sobre Educação em Computação, (pp. 493-502). Porto Alegre: SBC.

Oliveira, C. L. V . (2020) "JavaScript descomplicado : programação para a Web, IOT e dispositivos móveis" / Cláudio Luís Vieira Oliveira, Humberto Augusto Piovesana Zanetti. - São Paulo : Érica. 216 p.

Oliveira, Matheus Barbosa de; Rebouças, Ayla Débora Dantas de Souza (2021). Avaliando um Aplicativo Android Para Apoiar a Aplicação de Exercícios de Computação Desplugada. Revista Brasileira de Informática na Educação, [S.1.], v. 29, p. 798-826, ago. 2021. ISSN 2317-6121. Disponível em: <https://www.brie.org/pub/index.php/rbie/article/view/v29p798>. Acesso em: 08 set. 2021.

Organização Figma. Uma plataforma de design para escala. Disponível em:<https://www.figma.com/organization/>. Acesso em: 23 abr. 2021.

React Native. Aprenda uma vez, escreva em qualquer lugar. Disponível em: $<$ https://reactnative.dev/>. Acesso em: 18 abr. 2021.

Souza, Kellcia Rezende; KERBAUY, Maria Teresa Miceli (2017). Abordagem quantiqualitativa: superação da dicotoamia quantitativa-qualitativa na pesquisa em educação. Educação e Filosofia, v. 31, n. 61, p. 21-44,

UNICAMP. Computação Desplugada. Disponível em: <http://desplugada.ime.unicamp.br/>. Acesso em: 12 abr. 2021.

Veras, R. M.; Araujo, F. H.; Silva, R. R.; de Paula Jr, I. C. (2010) "Ferramenta Computacional para o Ensino de Algoritmos de Ordenação". In Anais do Simpósio Brasileiro de Informática na Educação - SBIE 2010.

Wangenheim, C. G. von; Araújo, G.; de Medeiros, S.; Missfeldt Filho, R.; Petri, G.; da Cruz Pinheiro, F.; \& Hauck, J. C. (2019) "Desenvolvimento e Avaliação de um Jogo de Tabuleiro para Ensinar o Conceito de Algoritmos na Educação Básica”. Revista Brasileira de Informática na Educação - RBIE. RBIE V.27, N.3 - 2019 (ISSN online: 2317-6121; print: 1414-5685).

Werlich, C.; Torrá, L. F.; Crema, C.; Gasparini, I.; Kemczinski, A. (2019). Objetos de Aprendizagem Plugados e Desplugados: um estudo de caso com ordenação alfabética. In: Anais do XXV Workshop de Informática na Escola. SBC, 2019. p. 49-58. 